\title{
Leveraging the Electronic Health Record to Get Value from Referrals
}

\author{
Michael K. Poku ${ }^{1}$ Nima A. Behkami ${ }^{2}$ David W. Bates 3,4
}

${ }^{1}$ Department of Medicine, Johns Hopkins Hospital, Baltimore, Maryland, United States

2 Strategy and Commercial Model Innovation, Merck \& Co., Philadelphia, Pennsylvania, United States

${ }^{3}$ Division of General Internal Medicine and Primary Care, Department of Medicine, Brigham and Women's Hospital, Boston, Massachusetts, United States

${ }^{4}$ Department of Health Policy and Management, Harvard T. H. Chan School of Public Health, Boston, Massachusetts, United States

Appl Clin Inform 2017;8:1054-1056.

Healthcare costs in the United States continue to climb as we proceed to transition away from a fee-for-service system of care to value-based care delivery. The principal aim of this delivery transformation toward value is to maximize patient-centered outcomes at the lowest possible cost. For instance, a key aspect of the thesis underlying the Health Information Technology for Economic and Clinical Health (HITECH) Act-an important piece of legislation driving the transition-is that incorporating electronic health records (EHRs) and other information technologies into the workflow of the healthcare delivery system stands to enable population health management and bring about enhanced value. The HITECH Act, in addition to encouraging the spread of EHRs to unprecedented levels, has led to the digitization of physician order entry, paved the way for broad patient electronic access, and spurred providers to send data to immunization registries and public health agencies. Despite these strides, there have been no formal efforts at the federal level to improve the referral process by leveraging EHRs and other digital information systems. We know that the careful management of the referral process is an important element in terms of delivering high-value care. ${ }^{1}$ We posit that EHRs can further drive transformational value if they deliver the referral decision support providers need.

The referral process influences a significant portion of healthcare spending. Specific referral behaviors are dictated by providers' preferences and practice patterns much like other tests and orders controlled by providers. ${ }^{2}$ Referrals directly impact the quality of healthcare delivered. While appropriate referrals to cost-efficient specialists can enhance care quality, overuse of referrals or referrals to less meticulous providers can lead to increased spending without a return in value. Thus, a thoughtful and concerted effort centered on
Address for correspondence Michael K. Poku, MD, MBA, 1800 Orleans Street, Baltimore, MD, 21287, United States (e-mail: Michael.Poku@jhmi.edu).

driving value through optimizing the referral process has the potential to improve the delivery of healthcare services. This is especially true given the growing number of referrals. The proportion of ambulatory visits resulting in a referral has increased substantially over time, doubling from 1999 to 2009. ${ }^{3}$ Trends and best practices related to provider referrals are largely understudied, but there is significant variability in referral practices across the United States. ${ }^{4}$ Consequently, improvement of referral processes represents a key potential source of value creation, particularly as this process relates to primary care providers who stand at the forefront of referrals. In fact, early savings among Massachusetts ACOs were largely ascribed to changing referral patterns to low-cost specialists. ${ }^{1}$

Traditionally, primary care providers refer their patients to specialty providers within their organization (if they are part of a multispecialty organization or academic health system), or to providers selected based on a primary care provider's subjective sense of a specialist, or, worse yet, referrals are made on a more arbitrary basis to the first available specialist provider in the area who accepts the patient's insurance. A third of the healthcare spending in the United States may be waste (i.e., unnecessary and/or inefficient and/or overpriced) and the largely haphazard approach to specialty referrals is likely contributing. ${ }^{5}$

Success in managing total costs of care will depend on making good referral choices, and this in turn suggests that primary care providers may want to use "smart" digital tools embedded in the EHR (the centerpiece of their current workflow) to assist them in making referrals. To maximize effect, such a solution does not necessarily need to be built by EHR providers, but their functionality ought to be contained within these systems given that they already host a bulk of physician actions. Digital solutions that exist outside of the EHR will be received

August 23, 2017

accepted after revision

September 2, 2017
Copyright @ 2017 Schattauer
DOI https://doi.org/ 10.4338/ACI-2017-04-IE0066 .

ISSN 1869-0327. 
cumbersome for providers to adopt, given how closely their workflows are tied to the EHR interface. As the reimbursement system continues to migrate toward paying providers for value (i.e., outcomes deflated by total costs), such an analytical tool will be hotly demanded and buy in from primary care providers will occur naturally even if there is an initial learning curve that impairs provider efficiency temporarily. EMR vendors would compete with one another on functionality and price to win market share and features would be consistently updated to remain competitive. An analytical solution conveying relative cost and quality for individual specialists in a primary care provider's catchment area would be a muchneeded advance, as would institution-based guidelines for which referrals are or are not likely to deliver value. But costs are notoriously hard to assess in healthcare. If one goes to the supermarket, the prices for food are transparent. In contrast, the costs associated with even common procedures have been very hard for institutions to determine, despite laws in many states trying to improve transparency. ${ }^{6}$ In order for digital referral support tools to be useful, they must incorporate accurate costs of care (i.e., the total liability for the cost of care owed by patient and/or third-party payer). The success and failure of such tools can be tallied by assessing changes to referral patterns after solution implementation, including the associated total costs of care for a given population of patients.

An early example of a digital referral support-related solution has been the use of physician report cards by the states of New York and Pennsylvania for cardiac surgery. In the early 1990s, the states of New York and Pennsylvania began publicly releasing surgeon-specific data on coronary artery bypass surgery. There was a notable decrease in in-hospital mortality in both states soon after the introduction of the public-reporting system, and in New York lower performing surgeons were more likely to move clinical locations or switch careers altogether. ${ }^{7}$ However, despite encouraging results, surveys in 1997 and 2001 revealed that most cardiologists neither relied on the surgeonspecific data in making their referrals to surgeons nor did they share these data with patients. More recent examples of report card-like solutions include the Centers for Medicare and Medicaid Services' Physician Compare and Hospital Compare solutions, which publicly reports quality data. Unfortunately, evidence suggests that so far, these datasets are not routinely utilized by primary care providers. ${ }^{8}$ Ideally, digital decision aids to optimize the referral process should be embedded directly into primary care providers' workflow within existing EHRs. Such a decision aid would need to be supported by a database compiled of provider-level cost and quality data from all major third-party payers in a catchment area; the data could be organized by location/region, medical condition, certain patient characteristics, and/or by providers who accepted certain insurance carriers. With the advent of narrow networks (i.e., healthcare coverage plans with a limited number of providers in a given catchment area), it will be especially important that the list of in-network providers for a given patient be kept up to date in order for such a tool to truly add value clinically.

A potential barrier to implementing such referral management solutions is the fact that some primary care providers argue that such referral decision-support tools would have no impact in an age where much of the American population selfrefer themselves to many specialists without provider input. However, this should change under value-based care with benefit plans and provider reimbursement aligned to enhance healthcare value. Patients will benefit if they can be guided to low-cost, high-quality specialty providers.

Another barrier in implementing EHR provider referral support solutions may be establishing a common understanding of the specific healthcare costs specialists ought to be accountable for, in addition to understanding the associated quality metrics and appropriate length for a given episode of care. The Centers for Medicare and Medicaid Services has historically defined surgical procedures in episodes of care up to 90 days postprocedure with various associated quality measures (e.g., readmission and complication rates). Adhering to these, episode lengths and quality measures and focusing support tools to optimize surgical procedures would be a pragmatic initial approach. Care should also be taken to exclude costs associated with catastrophic illness clearly unrelated to the surgical procedure in question.

Another major concern is risk adjustment. The best specialists often care for the sickest patients. Without considering the many factors that influence patients' healthcare costs and outcomes (e.g., health status, socioeconomic status, and demographic information), primary care providers could easily utilize referral support tool data to arrive at erroneous conclusions; or worse yet, specialists may limit access to certain patients who might threaten their quality or cost performance. There are various acceptable, albeit imperfect, risk adjustment models that ought to be used in the proposed digital referral decision aids. The HHS-/CMS-HCC risk adjustment model has served as the basis of Medicare Part $\mathrm{C}$ and Part $D$ reimbursement for many years. This model and others should be implemented within provider referral support solutions and they should be updated and replaced as new advances in risk adjustment come along. ${ }^{9}, 10$

Despite these issues, we believe that implementation of digital provider referral support tools within now widely adopted EHRs will be a key innovation for improving the delivery system. Such tools need to be built and then used in concert with other innovations and solutions aimed at enhancing the referral process like digitally facilitated provider-to-provider "curbside" visits and telemedicine consults. Overall, referrals have not yet received enough attention in the drive to achieving value-based care. If the right incentives are implemented, providers will realize the potential enhanced referral management tools will have on their performance and they will demand and then implement these solutions, though there will clearly be bumps along this road.

\section{Multiple Choice Question}

What has been the trend of the proportion of ambulatory visits resulting in a referral from 1999 to 2009?
A. Doubled.
B. Decrease by a factor of 3 . 
C. Halved.

D. Remained constant.

Correct Answer: The correct answer is A, doubled.

Protection of Human and Animal Subjects

No human subjects were involved in this article.

\section{Conflict of Interest}

Dr. Poku and and Dr. Behkami have no conflicts to report. Dr. Bates consults for EarlySense, which makes patient safety monitoring systems. He receives equity and cash compensation from QPID, Inc, a company focused on intelligence systems for electronic health records. He receives cash compensation from CDI (Negev), Ltd, which is a not-forprofit incubator for health IT startups. He receives equity from Enelgy which makes software to support evidencebased clinical decisions. He receives equity from Ethosmart which makes software to help patients with chronic diseases. He receives equity from Intensix which makes software to support clinical decision making in intensive care. He receives equity from MDClone which takes clinical data and produces de-identified versions of it. Dr. Bates' financial interests have been reviewed by Brigham and Women's Hospital and Partners HealthCare in accordance with their institutional policies.

\section{References}

1 Song Z, Sequist TD, Barnett ML. Patient referrals: a linchpin for increasing the value of care. JAMA 2014;312(06):597-598

2 Gottlieb DJ, Zhou W, Song Y, Andrews KG, Skinner JS, Sutherland JM. Prices don't drive regional Medicare spending variations. Health Aff (Millwood) 2010;29(03):537-543

3 Barnett ML, Song Z, Landon BE. Trends in physician referrals in the United States, 1999-2009. Arch Intern Med 2012;172(02): 163-170

4 Choudhry NK, Liao JM, Detsky AS. Selecting a specialist: adding evidence to the clinical practice of making referrals. JAMA 2014; 312(18):1861-1862

5 Institute of Medicine of the National Academies. Best Care at Lower Cost: The Path to Continuously Learning Health Care in America. Institute of Medicine. Available at: http://www.nationalacademies. org/hmd/Reports/2012/Best-Care-at-Lower-Cost-The-Path-to-Continuously-Learning-Health-Care-in-America.aspx. Accessed January 22, 2017

6 Farrell KS, Finocchio LJ, Trivedi AN, Mehrotra A. Does price transparency legislation allow the uninsured to shop for care? J Gen Intern Med 2010;25(02):110-114

$7 \mathrm{Xi}$ AS. The next generation of physician report cards. AMA J Ethics 2015;17(07):647-650

8 Morsi E, Lindenauer PK, Rothberg MB. Primary care physicians' use of publicly reported quality data in hospital referral decisions. J Hosp Med 2012;7(05):370-375

9 Rose S. A machine learning framework for plan payment risk adjustment. Health Serv Res 2016;51(06):2358-2374

10 Glance LG, Kellermann AL, Osler TM, Li Y, Li W, Dick AW. Impact of risk adjustment for socioeconomic status on risk-adjusted surgical readmission rates. Ann Surg 2016;263(04):698-704 